\title{
Distorted-wave Born theory for electron capture during resonant nuclear scattering
}

\author{
D H Jakubaßa-Amundsen \\ Sektion Physik, Universität München, 8046 Garching, West Germany
}

Received 5 August 1987

\begin{abstract}
A quantum mechanical formulation of the distorted-wave Born theory is presented, taking full account of the internuclear potential. Upon expansion in terms of one of the two electronic potentials, the strong-potential Born theory is rederived. Thereby an additional term emerges which had not been considered previously and which has considerable influence on the capture probability at large scattering angles.
\end{abstract}

The distorted-wave Born (DWB) theory as introduced by Taulbjerg and Briggs (1983) gives a consistent description of charge transfer in energetic near-symmetric ion-atom collisions provided that sufficient care is taken to avoid divergencies (Macek 1987) which emerge in the case of Coulomb potentials (Dewangan and Eichler 1985). The DWB theory consists of the first term in an expansion of the exact scattering amplitude in terms of a distortion potential, which is defined through the choice of strong-potential Born wavefunctions as distorted waves in the initial and final channels (Burgdörfer and Taulbjerg 1986). By means of construction, the DWB theory is symmetric with respect to the two electron-nucleus potentials. A first-order expansion in one of the potentials yields the familiar strong-potential Born (SPB) approximation (Macek and Shakeshaft 1980, Jakubaßa-Amundsen and Amundsen 1980) which has been successfully applied to asymmetric collision systems (Macek and Alston 1982, Amundsen and Jakubaßa-Amundsen 1984a, McGuire and Sil 1983).

So far, the internuclear potential has not been taken into account in the DwB formulation. However, when charge transfer occurs in a reaction where the projectile and target nuclei form a compound system with a decay time of the order of the inverse energy transfer to the active electron, the internuclear potential must explicitly be included in a correct description. It has been taken into consideration in a quantum mechanical formulation of the SPB theory (Amundsen and Jakubaßa-Amundsen 1984b, Jakubaßa-Amundsen and Amundsen 1985), and structures in the capture probability were predicted in the case of resonant nuclear scattering. Subsequent experiments verified these predictions (Scheurer et al 1985, Horsdal et al 1986); however, some discrepancies between theory and experiment at large scattering angles remained unexplained.

In this letter we present another derivation of the quantum mechanical SPB theory by first extending the DBW formulation to include the internuclear potential $V_{N}$ to all orders. For the sake of simplicity, we restrict ourselves to the case of a pure three-body problem and consider the transfer of a target electron into a bound state of the projectile.

0022-3700/87/210705+05\$02.50 (C) 1987 IOP Publishing Ltd $\quad$ L705 
For rearrangement collisions, the Hamiltonian $H$ is split differently in the entrance and exit channel

$$
H=T_{\mathrm{N}}+V_{\mathrm{N}}+T_{\mathrm{e}}+V_{\mathrm{T}}+V_{\mathrm{P}}=H_{i}+V_{i}=H_{f}+V_{f}
$$

where $T_{\mathrm{N}}$ and $T_{\mathrm{e}}$ are the kinetic energies of the relative nuclear motion and of the electron, respectively, while $V_{\mathrm{P}}$ and $V_{\mathrm{T}}$ denote the potential of the electron in the projectile and target field, respectively. The distorted waves $\chi_{i}^{(+)}$or $\chi_{f}^{(-)}$in the entrance or exit channel are taken as eigenfunctions to $H_{i}$ or $H_{f}$, respectively. In close analogy to Burgdörfer and Taulbjerg (1986) we choose

$$
\begin{array}{ll}
H_{i}=H-V_{\mathrm{T}} G_{\mathrm{N}}^{(+)} V_{\mathrm{P}} & V_{i}=V_{\mathrm{T}} G_{\mathrm{N}}^{(+)} V_{\mathrm{P}} \\
H_{f}=H-V_{\mathrm{P}} G_{\mathrm{N}}^{(-)} V_{\mathrm{T}} & V_{f}=V_{\mathrm{P}} G_{\mathrm{N}}^{(-)} V_{\mathrm{T}}
\end{array}
$$

where the Green function $G_{A}^{( \pm)}$for the potential $V_{A}(A=\mathrm{N}, \mathrm{P}, \mathrm{T})$ is defined by

$$
G_{A}^{( \pm)}=\left[E-\left(T_{\mathrm{N}}+T_{\mathrm{e}}+V_{A}\right) \pm \mathrm{i} \varepsilon\right]^{-1} \quad \varepsilon \rightarrow 0
$$

$E$ being the total energy of the system (including the energy of the internuclear motion). Note that $H_{i}$ and $H_{f}$ are not Hermitian.

The strong-potential Born functions are defined in the following way (JakubaßaAmundsen and Amundsen 1985)

$$
\begin{aligned}
& \chi_{i}^{(+)}=\left(1+G_{\mathrm{NP}}^{(+)} V_{\mathrm{P}}\right)\left(1+G_{\mathrm{NT}}^{(+)} V_{\mathrm{N}}\right) \phi_{i}=\left(1+G_{\mathrm{NP}}^{(+)} V_{\mathrm{P}}\right) \psi_{i \mathrm{~N}} \\
& \chi_{f}^{(-)}=\left(1+G_{\mathrm{NT}}^{(-)} V_{\mathrm{T}}\right)\left(1+G_{\mathrm{NP}}^{(-)} V_{\mathrm{N}}\right) \phi_{f}=\left(1+G_{\mathrm{NT}}^{(-)} V_{\mathrm{T}}\right) \psi_{f \mathrm{~N}} .
\end{aligned}
$$

In these expressions, $\phi_{i}$ is the eigenfunction to $T_{\mathrm{N}}+T_{\mathrm{e}}+V_{\mathrm{T}}$ and $\phi_{f}$ is the eigenfunction to $T_{\mathrm{N}}+T_{\mathrm{e}}+V_{\mathrm{P}}$, while $\psi_{i \mathrm{~N}}$ and $\psi_{f \mathrm{~N}}$ in addition include the internuclear potential $V_{\mathrm{N}}$. The Green function $G_{A B}^{( \pm)}$contains the potential $V_{A}+V_{B}$.

It can easily be verified that $\chi_{i}^{(+)}$indeed is the eigenfunction to $H_{i}$. To this aim, the solution to $H_{i}$ is expressed by means of the Lippmann-Schwinger equation in terms of $\psi_{i \mathrm{~N}}$ :

$$
\chi_{i}^{(+)}=\psi_{i \mathrm{~N}}+G_{i}^{(+)}\left(V_{\mathrm{P}}-V_{\mathrm{T}} G_{\mathrm{N}}^{(+)} V_{\mathrm{P}}\right) \psi_{i \mathrm{~N}}
$$

where $G_{i}^{(+)}$is the Green function to $H_{i}$. With the help of appropriate propagator relations (such as $G=G_{0}+G_{0} V G$ for $H=H_{0}+V$ ), $G_{i}^{(+)}$can be written in the following way

$$
\begin{aligned}
G_{i}^{(+)} & =G_{\mathrm{NP}}^{(+)}+G_{i}^{(+)}\left(V_{\mathrm{T}}-V_{\mathrm{T}} G_{\mathrm{N}}^{(+)} V_{\mathrm{P}}\right) G_{\mathrm{NP}}^{(+)} \\
& =G_{\mathrm{NP}}^{(+)}+G_{i}^{(+)} V_{\mathrm{T}} G_{\mathrm{N}}^{(+)} .
\end{aligned}
$$

Upon insertion into the term $G_{i}^{(+)} V_{\mathrm{P}} \psi_{i \mathrm{~N}}$ of (5), the SPB expression (4) is recovered. The proof for $\chi_{f}^{(-)}$proceeds in a similar way.

The exact transition amplitude for electron capture is given by (Joachain 1975)

$$
W_{f i}=\left\langle\chi_{f}^{(-)}\left|V_{f}^{+}\right| \Psi_{i}^{(+)}\right\rangle+\left\langle\chi_{f}^{(-)}\left|V_{i 0}-V_{f}^{+}\right| \phi_{i}\right\rangle
$$

where $V_{i 0}=V_{\mathrm{N}}+V_{\mathrm{P}}$ is the asymptotic perturbation in the initial channel. The second term in $(7)$ is the surface term which accounts for the transfer induced by the final distortion $V_{f}-V_{f 0}$ (with $V_{f 0}=V_{\mathrm{N}}+V_{\mathrm{T}}$ ). Replacing the exact three-body scattering state $\Psi_{i}^{(+)}$by the distorted wave $\chi_{i}^{(+)}$one arrives at the distorted-wave Born theory:

$$
W_{f i}^{\mathrm{DWB}}=\left\langle\chi_{f}^{(-)}\left|V_{\mathrm{T}} G_{\mathrm{N}}^{(+)} V_{\mathrm{P}}\right| \chi_{i}^{(+)}\right\rangle+\left\langle\psi_{f \mathrm{~N}}\left|V_{\mathrm{N}}+V_{\mathrm{P}}+V_{\mathrm{T}} G_{\mathrm{NT}}^{(+)} V_{\mathrm{N}}\right| \phi_{i}\right\rangle
$$

Thereby the surface term has been simplified by means of appropriate propagator relations. It can be shown that $W_{f i}^{\mathrm{DWB}}$ indeed is symmetric in the potentials $V_{\mathrm{P}}$ and $V_{\mathrm{T}}$. 
In the case of asymmetric systems, e.g. $Z_{\mathrm{P}} \ll Z_{\mathrm{T}}$ (where $Z_{\mathrm{P}}$ and $Z_{\mathrm{T}}$ are the charges of the projectile and target, respectively), one may expand $V_{\mathrm{T}} G_{\mathrm{N}}^{(+)} V_{\mathrm{P}}\left|\chi_{i}^{(+)}\right\rangle$in (8) up to first order in $V_{\mathrm{P}}$, which according to (4) corresponds to replacing $\chi_{i}^{(+)}$by $\psi_{i \mathrm{~N}}$. Then one arrives at the SPB approximation in its prior form, which can be written as a sum of three terms:

$$
\begin{aligned}
& W_{f i}^{\mathrm{SPB}}=W_{f i}^{(0)}+W_{f i}^{(1)}+W_{f i}^{(01)} \\
& W_{f i}^{(0)}=\left\langle\chi_{f}^{(-)}\left|V_{\mathrm{N}}\right| \phi_{i}\right\rangle \quad W_{f i}^{(1)}=\left\langle\chi_{f}^{(-)}\left|V_{\mathrm{P}}\right| \psi_{i \mathrm{~N}}\right\rangle \\
& W_{f i}^{(01)}=\left\langle\psi_{f \mathrm{~N}}\left|V_{\mathrm{P}}\right| \psi_{i \mathrm{~N}}-\phi_{i}\right\rangle .
\end{aligned}
$$

The charge transfer induced by the recoiling nucleus is described by $W_{f i}^{(0)}$, while $W_{f i}^{(1)}$ denotes the capture mediated by the projectile field. In contrast to the two-potential formula for excitation, there appears in the case of rearrangement collisions a third term, $W_{f i}^{(01)}$, which is of Brinkman-Kramers type. It vanishes for $V_{\mathrm{N}}=0$ and may thus also be related to recoil; due to the presence of $V_{\mathrm{P}}$ it shows, however, a different dependence on scattering angle $\vartheta$ than $W_{f i}^{(0)}$. We would like to note that this term also emerges in the derivation of the SPB theory from the Faddeev equations; it has, however, been overlooked previously (Jakubaßa-Amundsen and Amundsen 1985).

Using the same techniques as in Jakubaßa-Amundsen and Amundsen (1985), $W_{f i}^{(01)}$ is found to have two contributions, a recoil term $W_{f i}^{R(01)}$ which survives in the semiclassical limit, i.e. when the nuclear scattering amplitude $f^{(+)}(K, \vartheta)$ varies slowly with energy on the scale of the energy transferred to the electron, plus a sticking term $W_{f i}^{S(01)}$ which is related to the inner part of the internuclear wavefunction and consequently describes transfer while the two nuclei stick together. Explicitly, one has in the case of pure Coulomb fields (in atomic units $\hbar=m=e=1$ ):

$$
\begin{gathered}
W_{f i}^{R(01)}=-\frac{\mathrm{i} Z_{\mathrm{P}}}{4 \pi^{2} \mu_{i}} f^{(+)}\left(K_{i}, \vartheta\right) \int \mathrm{d} \boldsymbol{q} \varphi_{i}^{\mathrm{T}}(\boldsymbol{q}) \hat{\varphi}_{f}^{*}\left(\boldsymbol{q}-\boldsymbol{v}_{f}\right) \\
\times\left(\pi \delta\left(\Delta E-v^{2} / 2+\boldsymbol{q} \boldsymbol{v}_{f}\right)+\frac{\mathrm{i}}{\Delta E-v^{2} / 2+\boldsymbol{q} \boldsymbol{v}_{f}}\right) \\
W_{f i}^{S(01)}=\frac{\mathrm{i} Z_{\mathrm{P}}}{4 \pi^{2} \mu_{i}}\left(f^{(+)}\left(K_{f}, \vartheta\right)-f^{(+)}\left(K_{i}, \vartheta\right)\right) \int \mathrm{d} \boldsymbol{q} \varphi_{f}^{P *}\left(\boldsymbol{q}-\boldsymbol{v}_{f}\right) \hat{\varphi}_{i}(\boldsymbol{q}) \\
\times\left(\pi \delta\left(\Delta E-v^{2} / 2+\boldsymbol{q} \boldsymbol{v}_{f}\right)-\frac{\mathrm{i}}{\Delta E-v^{2} / 2+\boldsymbol{q} v_{f}}\right) .
\end{gathered}
$$

In these expressions, $\varphi_{i}^{\mathrm{T}}(\boldsymbol{p})$ and $\varphi_{f}^{\mathrm{P}}(\boldsymbol{p})$ are the electronic target and projectile boundstate wavefunctions, respectively, in momentum space, and $\hat{\varphi}$ denotes the Fourier transform of the bound-state function $\varphi(\boldsymbol{r})$ multiplied by $1 / \boldsymbol{r}$. The energy difference between the final and initial electronic states is abbreviated by $\Delta E \equiv E_{f}^{\mathrm{P}}-E_{i}^{\top}, \boldsymbol{K}_{i}=\mu_{i} \boldsymbol{v}_{i}$ and $\boldsymbol{K}_{f}=\mu_{f} \boldsymbol{v}_{f}$ are the nuclear momenta and $\mu_{i}$ and $\mu_{f}$ are the reduced masses in the entrance and exit channels, respectively. Equations (10) and (11) is accurate up to the order of $m / M$ (the mass ratio between electron and nucleus) such that $v_{i}=v_{f}=v$ equals the beam velocity. Also, a weak $q$ dependence in the scattering amplitude has been neglected as well as a contribution to the sticking term resulting from the overlap of the internuclear wavefunction with a plane wave within the nuclear radius. In the case of hydrogenic wavefunctions, the expressions (10) and (11) can be reduced to one-dimensional integrals which are readily evaluated numerically. 
In order to investigate the influence of $W_{f i}^{(01)}$ on the capture amplitude, we have calculated electron capture from the carbon $\mathrm{K}$ shell by $1 \mathrm{MeV}$ protons into the ground state as a function of the scattering angle. As is evident from figure 1 , the inclusion of $W_{f i}^{(01)}$ causes a strong decrease of the capture probability at the larger scattering angles in contrast to a description where $W_{f i}^{(01)}$ is omitted. A comparison with experimental data from Horsdal Pedersen (1987) and Scheurer et al (1985) clearly favours the theory based on equation (9).

There is thus a strong indication that $W_{f i}^{(01)}$ should not be neglected in future large-angle SPB calculations. However, as concerns the numerical evaluation of $W_{f i}^{\mathrm{SPB}}$, a problem arises due to the fact that $W_{f i}^{(0)}+W_{f i}^{(1)}$ have hitherto been evaluated in full peaking approximation which seems (at least for $W_{f i}^{(1)}$ ) hard to relax, while $W_{f i}^{(01)}$ is calculated from (10) and (11) without approximation. Taken into consideration that both terms are roughly of the same magnitude but different in phase, the inaccuracy of the peaking approximation may be strongly enhanced. This is presumably the reason for some overestimation of the capture probability at the smaller scattering angles.

In conclusion, we have shown that a consistent derivation of the strong-potential Born theory from a quantum mechanical formulation of the distorted-wave Born theory leads in the case of a non-vanishing internuclear potential to an additional term which is also of first order in the weak electronic potential. This term should be included in the calculation of capture probabilities because it is of the same order of magnitude as the potential and recoil capture contributions considered previously. The result will be a decrease of the capture probability with scattering angle at large angles, in closer agreement with existing experimental data.

I should like to thank E Horsdal Pedersen for the communication of experimental

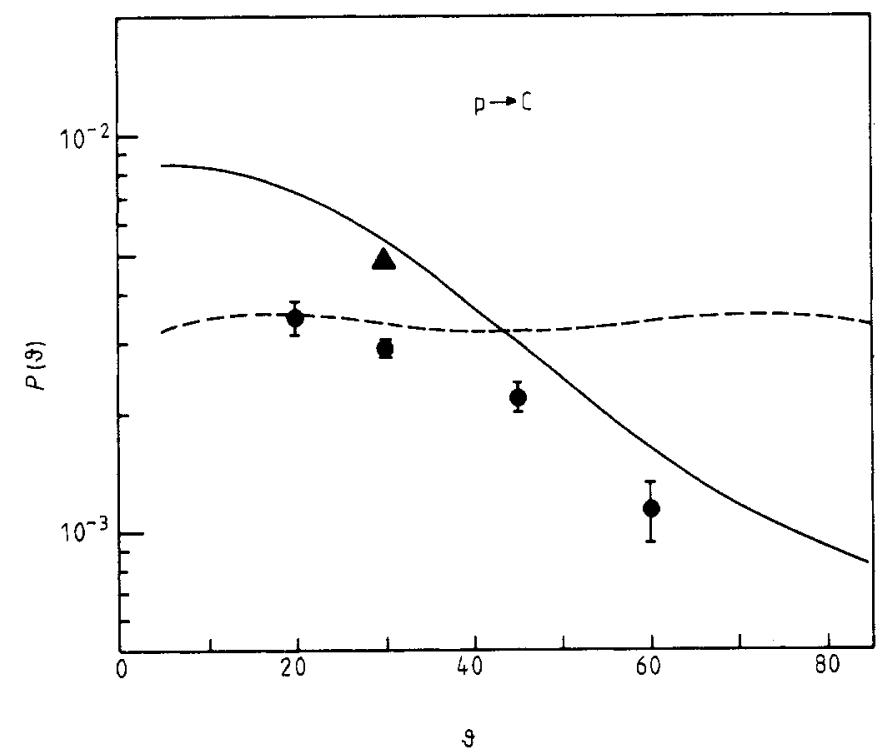

Figure 1. $\mathrm{K}-\mathrm{K}$ capture probability in $1 \mathrm{MeV} \mathrm{p}+\mathrm{C}$ collisions as a function of scattering angle $\vartheta$. The full curve denotes a calculation with the inclusion of $W_{f i}^{(01)}$, the broken curve one without $W_{f i}^{(01)}$. Comparison is made with experiments for $\mathrm{p}+\mathrm{CH}_{4}: \Phi$, Horsdal Pedersen (1987); $\boldsymbol{\Delta}$, Schevrer et al (1985). 
results prior to publication and $\mathrm{P} A$ Amundsen for enlightening discussions. Support from GSI Darmstadt is gratefully acknowledged.

\section{References}

Amundsen P A and Jakubaßa.Amundsen D H 1984a J. Phys. B: At. Mol. Phys. 172671 - 1984b Phys. Rev. Lett. 53222

Burgdörfer J and Taulbjerg K 1986 Phys. Rev. A 332959

Dewangan D P and Eichler J 1985 J. Phys. B: At. Mol. Phys. 18 L65

Horsdal E, Jensen B and Nielsen K O 1986 Phys. Rev. Lett. 57675

Horsdal Pedersen E 1987 Nucl. Instrum. Methods B 24/25 130

Jakubaßa-Amundsen D H and Amundsen P A 1980 Z. Phys. A 297203

1985 J. Phys. B: At. Mol. Phys. 18757

Joachain C J 1975 Quantum Collision Theory (Amsterdam: North.Holland) p 448

Macek J 1987 Phys. Rev. A 36 in press

Macek J and Alston S 1982 Phys. Rev. A 26250

Macek J H and Shakeshaft R 1980 Phys. Rev. A 221441

McGuire J H and Sil N C 1983 Phys. Rev. A 283679

Scheurer J N, Baker O K and Meyerhof W E 1985 J. Phys. B: At. Mol. Phys. 18 L85

Taulbjerg K and Briggs J 1983 J. Phys. B: At. Mol. Phys. 163811 